\title{
Le retour de Pologne d'Henri III: images alexandrines du roi au Bucentaure ${ }^{1}$
}

GUY

POIRIER

Résumé: Si la véritable nature du roi Henri III, celui que Pierre Chevallier dénommait le roi shakespearien, demeure scellée pour l'éternité, les pièces de circonstance écrites pendant les premières années de son règne peuvent en revanche nous renseigner sur les tentatives parfois contradictoires de "mise en discours" de l'image du jeune souverain. L'analyse littéraire de ces oeuvres, jumelée à l'étude de leur fortune chez les chroniqueurs et historiens qui fixèrent les premiers la légende du roi, permet donc de saisir les soubresauts des vastes manipulations imagistiques qui bientôt entraîneront le dernier des Valois dans les méandres d'une légende infâme.

Drince de la Renaissance trop souvent malmené par la plume acerbe de ses contemporains, Henri III de France, le fils bien-aimé de Catherine de Médicis, ne fut pas mieux servi par la modernité. Les Romantiques ne ressuscitèrent-ils pas ainsi sa cour en liant les unes aux autres, sur fond de scène maniériste, des allusions grotesques grappillées ça et là chez les historiens, les mémorialistes et les pamphlétaires? N'en firent-ils rien de moins qu'une véritable figure d'inverti de carnaval? Heureusement, pourrions-nous croire, les historiens du vingtième siècle réservèrent un meilleur sort aux mânes de ce roi honni. Circonstances atténuantes, lettres, détresse psychologique et enquêtes comptables sur la cour furent tour à tour évoquées afin de retirer à ce roi son bilboquet pour mieux lui rendre le sceptre et la couronne.

Même si Pierre Chevallier affirmait encore en 1985 qu'Henri III aurait pu être "très bon prince, s'il eût rencontré un bon siècle,"2 le dernier des Valois devint, à la lumières des études de Pierre Champion, de Philippe Erlanger, de 
Pierre Chevallier et de Jacqueline Boucher, tantôt un héros national, "un prince de la paix et des arts," ${ }^{3}$ mort pour la France, ${ }^{4}$ ayant évité par l'assassinat des Guises une guerre de succession qui eût vraiment pu être désastreuse pour le royaume, tantôt un souverain obsédé par la stérilité de son union avec Louise de Lorraine qui cherche consolation et protection auprès des jeunes courtisans - ses mignons - l'entourant. ${ }^{5}$

Somme toute, Henri III, contrairement à l'image du roi esclave de ses passions que l'historiographie puis la littérature historique véhiculèrent, fut conscient de son rôle de chef d'État à la tête d'un royaume en proie aux guerres civiles et religieuses, et pourrait même faire figure de véritable Prince de la Renaissance, ayant su, malgré une situation politique qui ne s'y prêtait guère, réformer la cour ${ }^{6}$ et en faire l'instrument d'une nouvelle vitalité nationale. ${ }^{7}$

Il va sans dire qu'une telle entreprise de réécriture du passé déleste, dans son traitement des archives historiques, des documents écrits n'ayant désormais qu'une valeur anecdotique. Ces reliquats de la science historique ne sont pourtant pas inutiles: source précieuse pour le littéraire, leur mise en discours devient objet de prédilection pour les enquêtes sur l'imaginaire, ${ }^{8}$ au même titre que les pièces officielles qui s'attachaient à y répondre. En fait, les écrits polémiques ou dithyrambiques provenant de différents horizons politiques s'entrechoquèrent et entrèrent en dialogue, entraînés au sein d'une vaste constellation giratoire productrice de sens qui se mit en branle le moment propice venu, faisant feu de tout bois, des faits historiques comme des histoires tragiques ou prodigieuses.

Dans le cas bien précis de la description des moeurs du roi Henri III, une courte portion de sa vie peut même nous servir de laboratoire afin de démontrer l'origine du fonctionnement d'un tel système, dans la mesure où nous avons alors affaire à l'un des épisodes de sa biographie qui fut peut-être le plus souvent commenté par ses contemporains. Il s'agit en fait de la narration du retour de Pologne qui s'effectua à l'été 1574. Les étapes de la pérégrination de celui dont on se souvenait encore comme étant le grand duc d'Anjou reviennent sans cesse sous la plume de divers historiens mémorialistes des seizième et dixseptième siècles, de de Thou au plus obscur auteur protestant. Dans bien des cas, parailleurs, cet épisode narratif sert à développer une analyse psychologique du souverain. Tous semblent s'accorder, dans un premier temps, pour affirmer que le retour du roi trompa les attentes. En revanche, les causes expliquant cette déception varient d'un auteur à l'autre et sont habituellement associées à des événements bien précis qui se déroulèrent lors de son périple transalpin. 
Sans vouloir ici récrire l'histoire d'Henri III mais bien en analyser la mise en discours et en imaginaire des moeurs au seizième siècle, nous tenterons dans un premier temps de saisir, grâce aux travaux d'historiens qui nous sont contemporains, l'espace polémique dont la science historique s'était malencontreusement emparée, le confondant avec la réalité. Le matériau isolé, nous effectuerons alors une lecture des écrits officiels et polémiques qui décrirent le retour de Pologne et le séjour que le roi fit à Venise. Notre "réalité" sera donc celle du discursif et de l'imaginaire qui donna naissance, dès le début du règne du roi, à un intertexte se prêtant merveilleusement bien aux accusations morales.

C'est en fait Jacques-Auguste de Thou qui fut le premier à nous mettre la puce à l'oreille quant à l'importance symbolique de ces événements. Cet historien bien connu des chercheurs modernes, que l'on cite d'ailleurs fréquemment lorsque vient le temps d'offrir une description tant soit peu réaliste du roi, laisse sous-entendre, en décrivant le retour en France d'un Henri d'Anjou qui s'apprête à devenir roi, qu'une obscure transmutation s'est opérée:

L'entrée du Roi en France causa une étrange révolution dans tous les esprits.

Du vivant de Charles IX, personne ne paroissoit plus digne du trône que Henri, et tout le monde souhaitoit l'avoir pour maître; à peine fut-il arrivé, qu'on s'en dégoûta jusqu'à augurer fort mal de son règne. ${ }^{9}$

Partageant l'opinion des historiens de l'époque, de Thou laisse présager que le roi fut moins victime, lors de son retour en France, des événements — c'est-à-dire de la politique de Charles IX ou de la reine mère - que d'une modification profonde de sa personnalité qui se serait produite entre son départ pour la Pologne et la fin de son voyage en Italie. Ce mystère entourant les étapes du voyage du souverain cache pourtant, dans le portrait qu'en donne de Thou, une vérité qui demeurera incontournable pour un grand nombre de ses contemporains; Henri III devint " [ . . . ] le plus mal heureux de tous les Rois ses predecesseurs ..."10

Les historiens modernes se sont bien entendu penchés sur les raisons qui auraient pu expliquer cette transformation quasi maléfique et remontent habituellement au séjour en Pologne pour en trouver les traces. Jacqueline Boucher et Pierre Chevallier ont bien démontré que le roi ne s'y plut guère, et que des accusations ayant rapport à des actes contre nature surgirent sous la plume de ses détracteurs. Pierre Matthieu, ${ }^{11}$ ligueur, partisan sur le tard d'Henri IV, trace une esquisse des chocs culturels que semblent avoir vécu les Français ayant suivi le roi en Pologne. Selon lui, les moeurs civiles des Français importunaient et en fait étaient plutôt associées à des vices par les Polonais. En 
revanche, la renommée du roi entachée, il n'y avait qu'un pas à faire pour laisser entendre qu'il était dans sa nature de ne pas accorder les mêmes

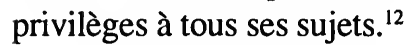

Pierre Matthieu donne également une brève description du comportement du roi en Pologne, description dont le ton rappelle les symptômes de la mélancolie:

Pour evaporer plus librement ses ennuys il paroissoit rarement en public, faisoit le malade, demeuroit quelquesfois quinze jours au lict, ne s'entretenant que de ce qu'il avoit laissé, ou esperoit revoir, n'avoit autre repos que celuy que la lassitude de se tourner et retourner luy apportoit. ${ }^{13}$

Matthieu, donnant la parole aux contemporains du roi, indique finalement que ces "indispositions" étaient par certains attribuées aux "angoisses de l'esprit" provoquées par intempérance des humeurs, ou encore par l'éloignement. ${ }^{14}$

Cependant, n'oublions pas que l'on soupçonne également le roi de souffrir d'une condition bien propre à son état de jeune prince. On dit notamment qu'il écrivait de Pologne à une princesse en utilisant son propre sang ${ }^{15}$ et qu'il aurait alors durement souffert d'être si éloigné de la présence féminine. À son retour, ce manque aurait fait place à un besoin irrépressible de leur présence continuelle, le détournant par là des affaires de l'État. ${ }^{16}$ L'expression officielle d'un tel état d'esprit apparaissait déjà sous le mode poétique dès 1573 , dans une pièce intitulée Adieu, du Roy de Pologne, au peuple François, et aux Dames de la Court; le roi fait ainsi ses adieux aux dames de Paris:

Mon coeur pourtant constant, quand le corps s'en ira,

Jamais (tant vous chérit) de vous ne partira,

Si qu'en Pologne estant, le coeur sera debvoir

De vous faire sans fin mon zelle apercevoir,

Qui tel est envers vous, à esté, et sera,

Qu'en toutte place et lieu secours vous donnera. ${ }^{17}$

Ce premier réseau d'allusions à l'attachement que le roi porte aux dames de la cour ne saurait être qu' inoffensif pour un jeune prince n'étant pas encore marié. De plus, l'éloignement et la froide Pologne suffisent en quelque sorte à contenir les débordements d'une mélancolie qui pourrait être avantageusement confondue avec un attachement au maniérisme purement français. La situation se complique évidemment lorsque le roi quitte la Pologne. Nous savons que le voyage, ou plutôt la fuite, s'effectua non pas par le chemin le plus court, mais bien en traversant les terres de Maximilien d'Autriche et de la République de Venise, où le futur souverain de France séjourna assez longtemps pour se faire désirer et ameuter les satiristes. 
Ladescription officielle du voyage octroyait, dès 1574 , une place déterminante aux constatations symboliques. La traduction d'un court ouvrage italien - écrit vraisemblablement par Rocco Beneditti et traduit chez Michel Jove - va ainsi dans ce sens. On insiste d'abord sur l'arc de triomphe élevé au roi à Venise: on y représente notamment la personnification des victoires du duc d'Anjou: “. . . avec un tel maintien, qu'il sembloit qu'elles voulussent en entrant couronner sa Majesté, prenant l'allusion aux quatre victoires gaignees par sa Majesté..." ${ }^{18} \mathrm{Ce}$ maintien et cette dignité font bien entendu figure de thème itératif encadrant l'épisode du Bucentaure. Nymphes et Victoires font alors place aux gallères, aux armées et à la joie - toujours fort digne - du souverain:

... le Bucintoro se mouvoit gravement, environné de tant de galleres, fustes, brigantins, et esquifz diversement ornez, et couvert d'infinité de banderolles de diverses couleurs, suivis d'une infinité de gondolles, et barques de toutes sortes, qu' il sembloient [sic] à voir, une grosse armee, ou une grande forest, au milieu des eauës. sa majesté remplie d'une joye indicible, dit, qu'il souhaitoit que la Royne sa mere se fust trouvee là presente. ${ }^{19}$

Quant aux bacchanales auxquelles le roi aurait assisté, elles n'apparaissent dans le discours officiel que sous la forme d'un banquet des plus raffinés; confitures rares et statues de femmes en sucre occupent l'espace sémantique de la courtisane. ${ }^{20} \mathrm{Il}$ faut dire par ailleurs que tout le symbolisme entourant l'entrée du roi ne peut que jeter le soupçon. On prévoit de Venise la joie des sujets, tout en insistant sur les exploits guerriers du prince alors qu'il était duc d'Anjou — ne négligeant à aucun prix les figures mythiques Pallas et Mars et soulignant la vertu d'un roi qui ne succombe pas aux "vains desirs," ces "plaisirs masquez" de la jeunesse. ${ }^{21}$

François de Mézeray, ce compilateur du dix-septième siècle, insistera sur la présence féminine en Vénétie, et rappellera le rôle des nymphes lors du fameux spectacle aquatique du Bucentaure:

Tout à l'entour de ce vaisseau flottoit un nombre infiny de gondoles: parmy lesquelles il y en avoit deux cents tapissées de riches étoffes, où paroissoient les plus belles Dames, toutes superbement parées; Spectacle qui eust pûdonner cette imagination aux Poëtes, que c'estoient les Nymphes, et les Graces, qui accouroient de toutes parts à la naissance de la mere des Amours. ${ }^{22}$

Quelques lignes plus loin, le lecteur n'est que peu surpris de constater que l'historien s'infiltre subrepticement dans la vie privée du roi: "Toutes les nuits il prenoit plaisir de visiter les plus belles, mesme les Courtisanes; et deux jours avant de partir il vid un bal de deux cens des plus aymables. .."23 
Le passage en Italie et la réception que l'on fit au roi à Venise marquent donc de façons différentes mais complémentaires les discours officiels et polémiques. Considérant les dates un peu tardives de certaines publications provenant de l'entourage du roi, on peut même se demander si elles ne visaient justement pas à faire taire certaines insinuations qui circulaient déjà.

Les historiens indiquent également que le roi reçut certains conseils lors de son séjour de Pologne, provenant parfois de l'Empereur Maximilien, de Damville rencontré à Turin ou des Vénitiens. Dans la plupart des cas, on aurait suggéré au roi de rétablir la paix au plus tôt. Scipion Dupleix, historien qui sera protégé par Marguerite de Valois, rapporte ainsi qu'un même conseil lui fut donné: “. . . ottroyer la paix à tous ses subjets, sans considerer la diversité des religions . .."24 La Popelinière, autre historien calviniste, rapporte également que l'Empereur aurait suggéré à Henri d'agir, à son retour en France, à l'instar d'un bon père de famille. ${ }^{25}$

Les conseils allégués par les historiens protestants devinrent rapidement choses du passé lors de l'arrivée en sol français, et ce pour des raisons diverses si l'on en juge par les opinions exprimées. La Popelinière rappelle d'abord, en solitaire, l'étrange changement d'attitude du nouveau souverain qui choisit de poursuivre l'effort de pacification armée entamée par son frère. ${ }^{26}$ Pour plusieurs, cependant, Henri fit dès son retour le jeu de sa mère et de certains conseillers. ${ }^{27}$ Surgit alors de nouveau l'ampleur de l'emprise du sexe féminin sur le roi. Ces deux types de commentaires se suivent effectivement dans bien des textes, comme si l'un pouvait expliquer l'autre, ou encore servir de cause ou d'effet. Mais reprenons la formulation de Mézeray. Immédiatement après avoir indiqué au lecteur que le roi avait remis aux bons soins de la reine mère le royaume, il note son retrait du regard de ses sujets et son avilissement auprès de femmes dans un lieu privé:

Si bien que luy laissant presque toute l'authorité, il paroissoit rarement en public, et demeuroit presque tousjours dans l'antichambre avec les Dames, ou dans le cabinet avec ses favorits; qui du commencement s' assujettissoient entierement à sa mere, et luy rendoient compte de toutes ses pensées. ${ }^{28}$

Pour Jean de Serres, qui suit toujours Montluc et s'inspire de Du Haillan, ${ }^{29}$ le roi avait abandonné la gouverne de l'État à la reine et à d'autres conseillers, car il était “. . . fort occupé à entretenir les dames, desquelles il avoit esté eslongné pres d'un an." ${ }^{30}$ Scipion Dupleix, en revanche, donne un portrait bien élaboré du sentiment amoureux qui s'empara alors du roi; l'historien établit un rapport intime entre le "feu d'Amour" qui embrase le roi et la remise en question de 
son autorité par ses sujets. Les paramètres de ce dernier réseau suivent en fait les règles du pouvoir, version antique. L'homme aimant trop ne saurait alors être digne d'un monarque:

Cependant le Roy commença de carresser les Dames: desquelles il s'estoit sevré pendant qu'il estoit parmi les Polonnois, nation plus severe que la nostre. Le feu d'Amour, comme le plus puissant, estouffant en luy tout ce qui restoit de la chaleur martiale (laquelle luy avoit acquis tant de reputation devant qu'il allât en Pologne) lon apperceut que toutes ses inclinations tendoient à chercher le repos et prendre ses plaisirs dans les delices de la France.

Ses deportemens mols et effeminés commencerent aussi-tost de ravaller l'estime qu'on avoit auparavant de luy: quoy que ses intentions touchant le gouvernement de son Estat fussent loüables et pieuses. ${ }^{31}$

Les tentatives du roi qui cherche à reprendre en main le royaume et surtout à "relever l'Autorité Royale"32 sont alors tournées en ridicule, laissant paraître un prince qui ne parvient qu'à s'appuyer sur un passé, celui de sa jeunesse et de son aventure en Pologne, qui ne rime plus à rien en sol français. Ses réformes de l'ordre de la cour, les levers, la remise des placets, les repas et même la tenue des courtisans à la messe tiennent de l'exercice de contrition. L'Estoile notera, dans son Journal, que le roi - sur les conseils de cet entourage, sur qui l'on fait porter tout l'odieux des premières décisions du roi et des règles nouvelles - s'avère plus sévère et moins communicatif envers la noblesse, ce qui le rendit fort étrange. ${ }^{33} \mathrm{Ce}$ sentiment d'étrangeté qui l'entoure peu à peu se concrétise dans l'Histoire universelle de de Thou à laquelle nous revenons. Le roi semble alors, sous la plume de l'historien, réactiver inutilement des épisodes de son passage à Venise. Ce morceau de bravoure rappelle effectivement le triomphe du Bucentaure:

... on le voyoit qu'enfermé avec quelques favoris dans un petit bateau peint, qui se promenoit sur la Saone; il ne mangeoit plus qu'avec une balustrade qui ne permettait pas de l'approcher; [. . . . La plus grande partie du jour il se renfermoit dans son cabinet avec quelques jeunes gens qui seuls avoient l'oreille du Prince, sans qu'on sçût par où ils étoient parvenus à cette distinction ... ${ }^{34}$

Cette scène, qui aurait pu frapper l'imagination des pamphlétaires, était néanmoins rapportées dans une brochure officielle intitulée: L'Ordre tenu à l'arrivee du Treschrestien Roy de France et de Pologne, Henry de Valois troisiesme de ce nom: 
Iceux avertis que sa Majesté passeroit la Saone, font faire à l'endroict de l'Arsenal un Bucentore en forme d'un Chasteau à la Venitienne [. . . ] sur le portail au devant se voit un Dieu Mars flamboyant en armes, et une Pallas tenant en sa main un Javelot. . . 35

De fait, cette tension entre la puissance virile du roi et une réalité moins brillante se laisse deviner dans les entrées et la poésie courtisane de la même époque. Blaise de Vigenère insistera sur la vertu guerrière d'Henri III, mais en rappelant ses exploits d'avant le séjour en Pologne, le prince adolescent “... n'ayant encore un seul poil de barbe." 36

D'autres pièces de circonstance permettent également de percevoir les parcours symboliques exploités par les historiens. Dans sa Bergerie touchant la mort du Roy Charles IX et l'heureuse venue de Henry III, Pierre de Monchault fait d'Henri III le sauveur qui ramènera en France le "bel aage," accompagné d'Astrée, la déesse de la justice:

Voicy dessoubs Henry revenir ce bel aage,

Qui soubs Saturne fut, quand le berger aux champs

Demeuroit nuict et jour sans crainte des meschans

La vertu aura lieu, on punira le vice,

Astree reviendra Deesse de Justice. ${ }^{37}$

Amadis Jamyn, dans le magnifique premier livre de ses Oeuvres poétiques également publié en 1575 et entièrement consacré au retour du roi en France et à la famille royale, ne dira pas autre chose lorsqu'il affirmera:

Voyci le jour heureux et sur tous desiré,

Que la France revoit de son Roy la presence:

Le Soleil devers nous pour mieux le voir s'avance,

Et l'honneur des vertus se tient plus asseuré. ${ }^{38}$

En revanche, il avait aussi laissé entendre, dans l'un des poèmes qui précédait cet extrait, que l'Italie, figure allégorique, s'appliqua à retenir le roi dans les rets de l'amour et du plaisir:

Ceste Italie ainsi belle et plaisante,

Dame amoureuse à vos yeux represente

Tous les plaisirs que l'art ingenieux

Peut inventer en la terre et aux cieux,

Voulant garder vostre royalle essence,

Et nous priver de telle jouïssance. ${ }^{39}$

Cette Italie-Calypso, usant des plaisirs de l'amour, n'abandonnera pas si aisément le roi à son ancienne patrie. Bien que le Soleil ravissant la lumière à 
l'Antarctique ${ }^{40}$ préside à son retour, bien que les images du renouveau se bousculent dans les premiers sonnets d'un Jamyn qui s'efforce vraisemblablement de louer un nouveau régime auquel on ne croit déjà plus, ${ }^{41}$ la Pologne mais surtout l'Italie - où le roi séjourna trop longtemps au goût de la reine mère - $s$ 'infiltrent dans les Mascarades offertes aux nouveaux mariés:

La France toute en voeus attend vostre venuë,

Comme une mere attend l'honneur de ses enfans,

Que trop long temps retient une terre inconnuë. ${ }^{42}$

Cette terra incognita, Henri la connaît en fait peut-être trop bien. La lecture du premier livre des Oeuvres poétiques de Jamyn nous livre un hymne où le roi est comparé à Alexandre le Grand. Nous savons qu'Alexandre était le nom que porta Henri de Valois du vivant de son père, mais peut-être est-il pour nous moins évident que son homonyme, le grand Alexandre, voulant conquérir les royaumes du Proche-Orient, dut aussi combattre et échapper aux vices de l'efféminement propre à ces contrées:

Cet Alexandre allant contre l'Asie

$\mathrm{Ne}$ rencontra que gens de molle vie,

Femmes plutost en hommes déguisez

Qu'hommes au fer de courage aiguisez,

Peints, parfumez, tous nourris en paresse

Et voluptez, pestes de la jeunesse ${ }^{43}$

Jamyn, d'après le contexte, fait vraisemblablement référence aux protestants que le duc d'Anjou combattit à La Rochelle avant son départ pour la Pologne, à moins qu'il ne faille interpréter à la lettre l'allusion au Levant et comprendre la Pologne ou Venise. N'oublions d'ailleurs pas qu'à cette époque l'étape ultime avant d'atteindre le royaume de la Grande Porte demeurait la République des Doges. Bien entendu, aucun combat n'y fut mené, et il faudrait peut-être plutôt croire que les tensions provoquées en Pologne purent prendre les allures d'une bataille quelconque. L'impact du séjour vénitien se calcule différemment: la lutte devient celle que doit livrer le roi contre la richesse et la volupté - et qu'il perdra selon les dires de la majorité des historiens - et c'est celle, ironiquement, que devront à leur tour soutenir les Réformés contre les mignons de l'Alexandre français.

Le recueil d'Amadis Jamyn est donc parsemé de clefs qui laissent entendre que l'avenir de la représentation imaginaire du roi se joue dès les premières années de son règne. L'historiographie n'aura au fond que peu à faire afin de relier les fragments allusifs ou biographiques véhiculés par les pièces officielles ou courtisanes. Avec Jamyn, l'ambiguïté semble même 
frôler la prophétie; à moins que son oeuvre ne trace inconsciemment les prémisses d'une coupure culturelle et épistémologique qui ne permettra bientôt plus au roi de participer aux fêtes de la cour ou de s'entourer de jeunes nobles sans encourir de jugements sévères quant à ses moeurs. Jugements, par ailleurs, qui ne naîtront pas innocemment; à son retour d'Italie, Henri III aurait été fortement impressionné par l'expérience mystique.

Ce dernier réseau d'images qui prendra peu à peu forme dans les écrits des mémorialistes est déclenché par la mort de la Princesse de Condé. Mézeray emprunte en fait une voie de service afin d'explorer l'obnubilation du roi par la religion et l'amour. C'est donc la mort de la princesse qui l'aurait poussé, selon l'historien, à transformer des sentiments amoureux trop inquiétants en véritable obsession. Mais suivons la métamorphose des sentiments du roi:

. . . celles qui se picquoient de faire triompher leur beauté d'un sujet si auguste, se servirent à l'envy de toutes les adresses et de tous les artifices que leur vaine gloire avoit jamais estudiez, pour attacher les inclinations de ce Prince; Et luy qui estoit de complexion fort tendre à estre blessé, recevoit tous ces traits avec plus ou moins de sentiment. ${ }^{44}$

La complexion du roi alors bien déterminée par les paramètres du sentiment amoureux et mélancolique ne peut que servir de riche terroir à un premier excès religieux lors de la mort d'une bien-aimée. Le roi, comme pour une mauvaise répétition, franchit les barrières de la norme et sombre dans l'expression d'un maniérisme exacerbé, alors qu'il s'entoure d'objets funèbres. ${ }^{45}$ Cette expression morbide de sa passion disparaîtra au bout d'un certain temps, Mézeray soulignant que le roi en eut honte. ${ }^{46}$

C'est lors de la narration du voyage à Avignon que se déploie un réseau d'images mystiques qui liera pour de bon le roi à la pratique des processions et aux confréries, exercices de nouveau introduits en France sous l'inspiration de la pastorale du Concile de Trente et des Jésuites. Ces marques de dévotion, à leur début, ne semblaient trop plaire aux courtisans ... Scipion Dupleix indique que

Durant le sejour que le Roy fit à Avignon (où il y a diverses confrairies de Penitens) il s'enrolla en celle des Blancs, comme firent aussi Monsieur, le Roy de Navarre, et la plupart des Courtisans, avec moins de devotion que de complaisance. ${ }^{47}$

Il faut également préciser que les pièces officielles traditionnellement imprimées pour les entrées, les couronnements et les mariages demeurent ancrées, du moins pour les premières années du règne, dans une thématique historico-mythologique et biblique. Alexandre le Grand, Jules César et David, 
personnages ayant su rétablir l'ordre, sont évoqués. ${ }^{48} \mathrm{Il}$ y a, en revanche, une présence assez marquée du Saint-Esprit et de Dieu dans une première salutation de 1575. Les deux derniers vers de cette pièce de Jean-Antoine de Baïf illustrent bien la juxtaposition des images mythologiques — ou même romanesques dans le cas de l'Astrée - et du tableau désespéré de la France qu'on y présente:

Mais, ô bon Dieu, si quelque offense
Reste encore dessus la France
A expier par nos douleurs:
Au Roy donne santé parfaite,
A fin que ta volonté soit faite
Par sa main trenche nos malheurs.
Donne à mon Roy seure conduite,
Et garde sa fidelle suite,
Et les chemins ren bien aisez:
Et fay qu'à son heureuse entree,
Tumbent devant la bonne Astree
Fureurs et troubles appaisez.

Si Dieu doit ainsi venir en aide au roi, il ne saurait apparaître que sous un aspect maniériste, au milieu des héros de l'Antiquité ou de personnages romanesques. $\mathrm{N}$ 'en était-il pas également ainsi, si l'on suit le récit qu'en firent les commentateurs, de l'affectation mystique que le roi démontra lors de la mort de Marie de Clèves?

S'il peut paraître curieux, dans un premier temps, de trouver un si grand nombre d'allusions à la vie et à la conduite morale du roi lors de son retour, cette symbolique matière de Pologne et de Venise ainsi contaminée par la subjectivité des pamphlétaires ou des ennemis du roi est devenue pour nous source de renseignements sur l'imaginaire d'une époque. Nous avons ainsi pu y saisir les réseaux sémantiques négociés par les polémistes, la façon dont ils les choisissaient ou les exploitaient de nouveau, les limites qui semblaient contraindre la parole. En revanche, et ce fut le cas dans les exemples que nous avons abordés dans le présent article, ces allusions ne sont pas créées selon les règles de la génération spontanée, mais bien grâce à des emprunts et à un dialogue avec le lyrisme courtisan ou les pièces officielles devant louer la grandeur du roi. Ces échanges - de thèmes, de mythes, d'anecdotes presque incestueux devraient en fait nous permettre d'aborder différemment la lecture des pièces satiriques ou même des pamphlets politiques qui viendront bientôt ternir une fois pour toutes l'image du Prince. S'il y a une certaine vérité 
dans les écrits polémiques imprimés sous le règne d'Henri III, elle réside moins dans ce reflet de la réalité que dans l'emploi d'un imaginaire courtisan et lyrique en voie de métamorphose maniériste.

Ce qui s'avère le plus ironique, en revanche, c'est qu'Henri-Alexandre a peut-être lui-même donné le ton à la polémique. Dire l'exil de la Pologne, célébrer Venise et décrire l'entrée du roi à Lyon ne furent au fond qu'un premier exercice de style, bientôt repris - ou même précédé — par la plume mordante des satiristes. Première esquisse des isotopies qui nourriront la polémique des années quatre-vingt, ne sera-t-elle au fond constamment reconduite au cours du règne? Trompette du jugement dernier sonnant au rythme d'un retour dans sa patrie effectué à pas d'escargot, le Bucentaure, ce monstre biblico-mythique glissant sur la Saône, ${ }^{50}$ image du tréponème qu'il aurait contracté dans la République, cristallise autour de luil'accident primaire, la première bévue du règne: Alexandre a gagné la bataille du Levant, mais a rapporté d'Italie un butin facétieux recelant ces "voluptez, pestes de la jeunesse." 51

\section{Simon Fraser University}

\section{Notes}

1. Les recherches ayant conduit à la rédaction de cet article purent être effectuées grâce à une subvention ordinaire du Conseil de recherches en sciences humaines du Canada.

2. Pierre Chevallier, Henri III, roi shakespearien (Paris, Fayard, 1985), p. 17.

3. Pierre Champion, La jeunesse de Henri III (Paris, Grasset, 1942), p. 341.

4. Philippe Erlanger, Henri III (Paris, Gallimard, 1948), pp. 419-420; et Pierre Chevallier, Henri III, roi shakespearien, p. 706.

5. Ibid., pp. 16-17.

6. Jacqueline Boucher, “Société et mentalités autour de Henri III," thèse de doctorat, Université de Lyon, 1977, pp. 200 et 398.

7. Ibid., pp. 1534-1538.

8. Pour ces questions, consulter: Daniel-Henri Pageaux, Le Bûcher d'Hercule; Histoire, critique et théories littéraires (Paris, Honoré Champion, 1996).

9. Jacques-Auguste de Thou, Histoire universelle (Londres, 1734), p. 134.

10. “Ainsi donc ce Prince ayant esté heureux, glorieux et triomphant tant qu'il regnât, fut le plus mal-heureux de tous les Rois ses predecesseurs depuis qu'il revint de Pologne pour recueillir la succession de la Couronne de France." Scipion Dupleix, Histoire de Henry III (Paris, Claude Sonnius, 1630), p. 6. 
11. Gaston Dodu, Les Valois, histoire d'une maison royale (Paris, Hachette, 1934), p. 382.

12. “. . . les actions diverses et differentes des leurs estoient mocquees ou mesprisees: les courtoisies, les complimens, les façons plus civiles et polies leur estoient importunes: les vertus qui leur estoient inconnues estoient reputees vices estrangers: l'humeur de ce Prince estoit d'aymer quelqu'un par preference aux autres, et les Polonnois vouloient qu'il y eust en cela de l'egalité, et que ce Soleil ne demeurast pas plus en une autre maison du Zodiaque qu'en l'autre..." Pierre Matthieu, Histoire de France soubs les regnes de François l $^{\text {er }}$, Henri II, François II, Charles IX, Henri III, Henri IV, Louis XIII (Paris, Nicolas Buon, 1631), p. 326.

13. Pierre Matthieu, Histoire de France, p. 386.

14. “. . . les moins grossiers jugeoient bien que le corps feignoit d'estre malade pour donner quelque couverture aux angoisses de l'esprit, et que ceste indisposition venoit plus de l'ennuy du lieu, que de l'intemperance des humeurs. .." Id.

15. "Quand il escrivoit à une Princesse, il tiroit du sang de son doigt, et ouvroit et fermoit la picqueure à mesure qu'il falloit remplir la plume pour escrire. .." Id.

16. "La Royne mere, le Cardinal de Lorraine, les Ducs de Guyse, de Nevers, le Mareschal de Rets, le Chancelier de Birague, et quelques autres, tailloient et rongnoient les affaires à leur guise és secrets conseils du Cabinet. Le Roy leur en laissait volontiers toute la disposition, ententif seulement à courtiser les Dames, desquelles il avoit esté sequestré pres d'un an parmy ceste nation moins lascive que la nostre." Bernard de Girard, Seigneur du Haillan, Histoire generale des roys de France, t. II (Paris, Jean Petit-Pas, 1629), p. 528.

17. Jherosme le Jeune, Adieu, du Roy de Pologne, au peuple François, et aux Dames de la Court (Paris, Guillaume de Nyverd, 1573), fol. B.iiiv.

18. Rocco Benedetti, Discours des triomphes et resjouissances faicts par la Serenissime Seigneurie de Venise, à l'entrée heureuse de Henry de Valois (Lyon, Michel Jove, 1574), pp. 17-18.

19. Ibid., pp. 18-19.

20. “Aiant dancé assez d'espace de temps, fut donnee une collation à la Royalle en la salle de l'Election, où au lieu du tribunal du siege du Prince, estoit mise la chaire pour le Roy, et la table dressee pour sa Majesté estoit couverte de confitures rares, et tres exquises, et avec diverses statues de sucre: entre lesquelles estoit une femme, qui luy presentoit deux couronnes, lions, navires, griffons, et autres figures de diverses sftions [abbrév.: significations], excellemment faictes par la main de l'industrieux Nicolas de la Pigna. .." Ibid., pp. 34-35.

21. "A ta naissance tu promis au monde merveilles: sorti hors d'enfance tu as abondamment fourny tout ce que lon peut attendre d'une engeance royale. Mesmes en l'aage auquel les autres ont accoustumé s'esbattre à vains desirs, jettant arriere tels plaisirs masquez, tu te donnas à la suitte de Pallas et de Mars, dissipant tes ennemys de ton bras robuste et invaincu." Extraict d'un chant d'allegresse de Nohé Zambon Venitien, Sur la somptueuse et tres magnifique entree de Henry III. treschretien Roy de France, à Venise, à son retour de Pologne, en France (Lyon, Benoist Rigaud, 1574), fol. A.2. 
22. François de Mézeray, Histoire de France, t. III (Paris, Mathieu Guillemot, 1651), p. 27.

23. Ibid., p. 28.

24. Scipion Dupleix, Histoire de Henry III, p. 24.

25. “...l’Empereur Maximilian (qui luy persuadoient qu'embrassans à sa venuë tant inesperée, tous ses sujets comme le bon pere de famille fait ses enfans au retour d'un long et mallaisé voiage: [...]" La Popelinière, L'Histoire de France enrichie des plus notables occurances, t. II (Paris, Abraham H, 1581), fol $283^{r}$.

26. “... il changea d'avis, resolu sachans les armes levées du temps de son frere non encor mises bas: de poursuivre par toutes voies ceux qui ne voudroient poser les armes..." Id.

27. Pour le catholique — et frère de Jean Eudes - Mézeray: "Car quelque chose que Damville luy eust representée à Turin, il protesta à sa mère dès son arrivée qu'il ne tenoit la couronne que de ses soins, et qu'il vouloit dépendre de sa conduite plus absolument que jamais" (François de Mezeray, Histoire de France, t. III, pp. 34-35). Jean de Serres, qui deviendra l'historiographe d'Henri IV, rapporte quant à lui les propos de Montluc: "Il adjouste: le Roy arrivant à Lyon, à son entree on lui fit faire un erreur: car au lieu qu'il devoit assopir le tout, et nous donner la paix, qui estoit chose bien aisee lors, on le fit resouldre à la guerre" (Jean de Serres, Recueil des choses memorables advenues en France sous le regne de Henri II, François II, Charles IX, Henri III et Henri IV (Heden, 1603), p. 521.

28. François de Mézeray, Histoire de France, t. III, pp. 34-35.

29. Cf. supra n. 16.

30. Jean de Serres, Recueil des choses memorables, p. 521.

31. Scipion Dupleix, Histoire de Henry III, p. 33.

32. "Il ne parloit que de relever l'Autorité Royale aussi haut, que les Regnes precedensl'avoient voulu mettre bas. Tous ses discours n'estoient que de son voyage de Polongne, du sejour qu'il y avoit faict, des honneurs qu'on luy avoit rendu par tout, des perils et difficultez surmontees pour se rendre en son Royaume" (Pierre Matthieu, Histoire de France, p. 402).

33. "Ainsi sa Majesté cherchant tous moiens honnestes de pacifier son Roiaume, se resserra aussi d'autre costé au maniement des affaires de son Estat, se rendist plus severe et moins communicatif que les Rois ses predecesseurs, ce que la Noblesse n'estant accoustumée à telles façons, trouva fort estrange, (Aussi cela ne provenoit du Roy, mais d'un Conseil peu ami de la Noblesse de France); ne permettoit parler en mangeant, ne s'approcher de lui toutes personnes; neantmoins donnoit à certaines heures du jour audiance à tout le monde, mais ne respondoit requestes de ceux qui demandoient, ou se plaignoient de tors faits, s'ils n'estoient presens, et deduisoient leurs raisons par leur bouche (Pierre de L'Estoile, Registre-Journal du règne de Henri III t. I (Genève, Droz, 1992, p. 78).

34. Jacques-Auguste de Thou, Histoire universelle, p. 134.

35. L'Ordre tenu à l'arrivee du Treschrestien Roy de France et de Pologne, Henry de Valois troisiesme de ce nom (Lyon, Benoist Rigaud, 1574), p. 9.

36. Blaise de Vigenère, La Somptueuse et magnifique entree du Tres-chrestien Roy Henry III (Paris, Nicolas Cheneau, 1576), p. 26. 
37. Pierre de Monchault, Bergerie touchant la mort du Roy Charles IX et l'heureuse venue de Henry III (Paris, Jean de Lastre, 1575), fol. C.2r.

38. Amadis Jamyn, Les Oeuvres poetiques (Paris, Robert Estienne et Mamert Patisson, 1575), fol. $3^{v}$.

39. "Au Roy, sur son retour de Pologne," Ibid., fol. $2^{r}$.

40.

"Or pour le moins ici vous revoyant Sur l'horizon des François flamboyant, Tel qu'un Soleil qui ravist sa lumiere A l'Antarctique, à fin qu'il nous esclaire, Je chanteray vos honneurs infinis Et la Deesse à qui vous estes fils, Dont la prudence au besoin esprouvee Pour son triomphe a la France sauvée."

Ibid., fol. $2^{\mathrm{v}}$ et $3^{\mathrm{r}}$

41. "Mais comme on voit la pucelle douter En un jardin de royale plaisance,

Quelle des fleurs son poulce doit cueillir:

Ainsi je sens la langue me faillir, Tant le sujet est riche d'abondance."

Ibid., fol. $3^{v}$

42. Ibid., fol. $6^{\mathrm{r}}$.

43. Ibid., fol. $40^{\text {r. }}$

44. François de Mezeray, Histoire de France, t. III, p. 44.

45. Ibid., p. 45.

46. Ibid., p. 46.

47. Scipion Dupleix, Histoire de Henry III, p. 37.

48. Claude de Montiornal, Bref discours surle tresheureux advenement de nostre tres-chrestien Roy Henry III, de ce nom aujourd'huy regnant (Lyon, Michel Jove, 1574), fol. A.3v et A.4r.

49. Jean-Antoine de Baif, Premiere salutation au roy sur son avenement a la couronne de France (Paris, Frederic Morel, 1575), fol. 8r.

50. Rappelons le sens premier de Bucentaure: centaure à corps de taureau. Cf. Trésor de la langue française, t. 4 (Paris, Centre National de la Recherche Scientifique, 1975), p. 1045.

51. Cf. supra n. 43. 